\title{
Neutral and Oxidized Triisopropylsilyl End-Capped Oligothienoacenes: A Combined Electrochemical, Spectroscopic, and Theoretical Study
}

\author{
Juan Aragó, ${ }^{[a]}$ Pedro M. Viruela, ${ }^{[a]}$ Enrique Ortí, ${ }^{*[a]}$ Reyes Malavé Osuna, ${ }^{[b]}$ \\ Barbara Vercelli, ${ }^{[b]}$ Gianni Zotti, ${ }^{[b]}$ Víctor Hernández, ${ }^{*[c]}$ Juan T. López Navarrete, ${ }^{[c]}$ \\ John T. Henssler, ${ }^{[d]}$ Adam J. Matzger, ${ }^{[d]}$ Yoshitake Suzuki, $^{[\mathrm{e}]}$ and Shigehiro Yamaguchi ${ }^{[\mathrm{e}]}$
}

\begin{abstract}
This work presents an analysis of the structural, electrochemical, and optical properties of a family of triisopropylsilyl end-capped oligothienoacenes (TIPS-Tn-TIPS, $n=4-8$ ) by combining cyclic voltammetry, spectroscopic techniques, and quantum-chemical calculations. TIPS-Tn-TIPS compounds form stable radical cations, and dications are only obtained for the longest oligomers $(n=7$ and 8$)$. Oxidation leads to the quinoidization of the conjugated backbone, from which electrons are mainly extracted. The absorption and fluorescence spectra show partially resolved vibronic structures even
\end{abstract}

at room temperature, due to the rigid molecular geometry. Two well-resolved vibronic progressions are observed at low temperatures due to the vibronic coupling, with normal modes showing $\approx 480 \mathrm{~cm}^{-1}$. Optical absorption bands display remarkable bathochromic dispersion with the oligomer length, indicative of the extent of $\pi$ conjugation.

Keywords: density functional calculations - electrochemistry • fluorescence $\cdot$ oligothienoacenes $\cdot$ optical properties $\cdot$ spectroelectrochemistry wavenumbers of $\approx 1525$ and

The optical properties of the oxidized compounds are characterized by in situ UV/Vis/NIR spectroelectrochemistry. The radical cation species show two intense absorption bands emerging at energies lower than in the neutral compounds. The formation of the dication is only detected for the heptamer and the octamer, and shows a new band at intermediate energies. Optical data are interpreted with the help of density functional theory calculations performed at the B3LYP/6-31G** level, both for the neutral and the oxidized compounds.

\section{Introduction}

Instituto de Ciencia Molecular, Universidad de Valencia

Valencia 46980 (Spain)

Fax: (+34) 963543274

E-mail: enrique.orti@uv.es

[b] Dr. R. Malavé Osuna, Dr. B. Vercelli, Dr. G. Zotti Istituto CNR per l'Energetica e le Interfasi, c.o. Stati Uniti 4 35127 Padova (Italy)

[c] Prof. V. Hernández, Prof. Dr. J. T. López Navarrete Department of Physical Chemistry, University of Málaga 29071 Málaga (Spain)

Fax: $(+34) 952-132000$

E-mail: hernandez@uma.es teodomiro@uma.es

[d] Dr. J. T. Henssler, Prof. Dr. A. J. Matzger

Department of Chemistry and

the Macromolecular Science and Engineering Program University of Michigan, 930 North University

Ann Arbor, Michigan 48109-1055 (USA)

[e] Dr. Y. Suzuki, Prof. Dr. S. Yamaguchi

Department of Chemistry, Graduate School of Science Nagoya University, Furo Chikusa, 464-8602 Nagoya (Japan)

Supporting information for this article is available on the WWW under http://dx.doi.org/10.1002/chem.200903343.
Linearly fused $\pi$-conjugated compounds constitute an important class of organic semiconductors due to their application in thin-film organic electronic devices, such as organic field-effect transistors (OFETs). ${ }^{[1,2]}$ Their rigid $\pi$-conjugated skeleton, free from conformational disorder, is a decisive characteristic leading to unique electronic properties and densely packed solid-state structures. This packing allows for high charge-carrier mobilities. Among such fused $\pi$-conjugated compounds, pentacene is probably the most studied system. Pentacene has attracted great interest because it presents some of the highest hole mobilities reported for organic crystals. ${ }^{[3]}$ However, pentacene suffers from the disadvantage of being easily oxidized, and is only moderately stable in air or under illumination. In this context, linearly fused oligothiophenes, or all-anti oligothienoacenes, emerge as a promising new class of $\pi$-conjugated compounds that combine the rigid planarity and extended conjugation of acenes with the chemical stability of oligothiophenes. Oligothienoacenes exhibit larger HOMO-LUMO gaps than their 
respective acene partners, and display a higher environmental stability. ${ }^{[4,5]}$ Furthermore, they favor face-to-face $\pi$ stacked structures in the solid state, ${ }^{[5]}$ due to the lower number of $\mathrm{CH}-\pi$ interactions, thus facilitating the achievement of high charge-carrier mobilities. ${ }^{[6]}$ These characteristics make oligothienoacenes very attractive as stable semiconducting materials for their use in OFETs. Indeed, pentathienoacene has already been implemented as the active material in FETs, achieving mobilities as high as $0.045 \mathrm{~cm}^{2} \mathrm{~V}^{-1} \mathrm{~s}^{-1} \cdot{ }^{[7]}$

Only a limited number of linearly fused thiophenes have been reported to date, including short nonsubstituted oligothienoacenes (up to the pentamer), ${ }^{[8,9]}$ the dibenzo-annulated dithieno[3,2-b:2', $\left.3^{\prime}-\mathrm{d}\right]$ thiophene trimer, ${ }^{[10-12]}$ and the fully fused polythiophene. ${ }^{[13]}$ Recently, new synthetic strategies have been developed that allow for the synthesis of longer all-anti oligothienoacenes up to the octamer. Maztger et al. ${ }^{[5]}$ synthesized pentathienoacene and heptathienoacene, both nonsubstituted and incorporating terminal triisopropylsilyl (TIPS) groups (Figure 1), through the coupling of two brominated thienothiophenes (two fused rings) or two dithienothiophenes (three fused rings), respectively, through a sulfide bridge. Yamaguchi et al. ${ }^{[14]}$ reported a new synthetic route to obtain oligothienoacenes end-capped with TIPS groups with an even number of rings (tetramer, hexamer, and octamer in Figure 1). The solubilizing TIPS groups enhance their purification and solution processability.

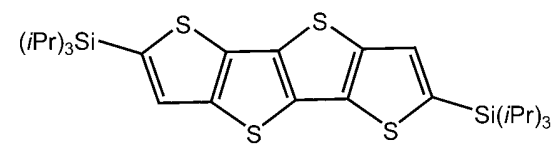

TIPS-T4-TIPS
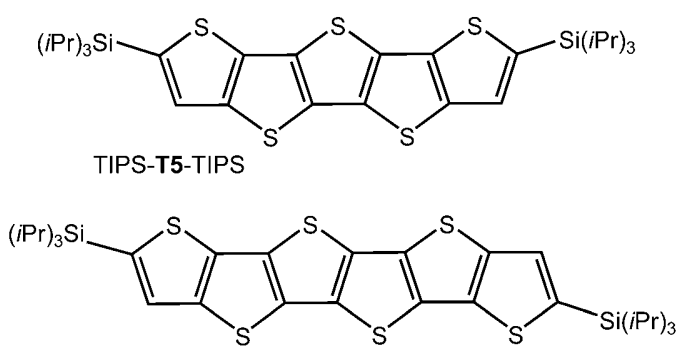

TIPS-T6-TIPS

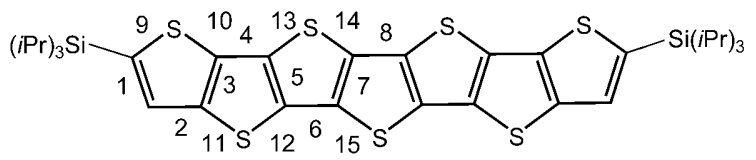

TIPS-T7-TIPS

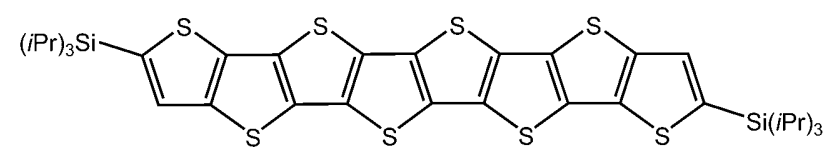

TIPS-T8-TIPS

Figure 1. Chemical structures of the triisopropylsilyl end-capped oligothienoacenes (TIPS-Tn-TIPS, TIPS: triisopropylsilyl) under study. The bond numbering used in the text is given for TIPS-T7-TIPS.
UV/Vis absorption and fluorescence spectroscopies have been found to be successful techniques for the investigation of the conformational disorder in $\pi$-conjugated compounds and, particularly, in oligothiophenes. ${ }^{[15]}$ Nonfused oligothiophenes present a high conformational disorder in the ground state due to the internal rotation around the interring $\mathrm{C}-\mathrm{C}$ bonds. In passing to the first excited state, they exhibit a reversal of the alternating single/double carboncarbon bond pattern (quinoidization) that hinders the free rotation around the inter-ring bonds and planarizes the molecule. ${ }^{[16,17]}$ These geometric changes have a large impact on the compounds' optical properties at room temperature, which show broad absorption bands and partially resolved vibronic fluorescence bands, as well as large Stokes' shifts. ${ }^{[18]}$ The conformational disorder decreases at low temperatures, leading to absorption and fluorescence bands with well-resolved vibronic structures. In contrast to oligothiophenes, oligothienoacenes are rigid systems with a low degree of conformational disorder. This fact produces unique optical properties compared to their nonfused homologues, and well-resolved absorption and fluorescence bands are observed at room temperature for oligothienoacenes. ${ }^{[5,14]}$

The charged species formed in the oxidation/reduction processes of a great variety of oligothiophenes have been monitored and characterized by using UV/Vis/NIR spectroelectrochemistry. ${ }^{[19-31]}$ Quantum-chemical calculations have been shown to be very helpful in correctly assigning the optical absorptions of the charged species, and in corroborating the nature of these species (radical cations, dications, and radical cation $\pi$-dimers) $)^{[32-38]}$ Therefore, the combination of spectroelectrochemistry and quantum-chemical calculations provides an efficient tool for the investigation of the charged species involved in the charge transport process in $\pi$-conjugated oligomers. Although numerous studies on the optical properties of both neutral and oxidized oligothiophenes have been reported to date, oxidized oligothienoacenes have received surprisingly little attention. Therefore, a comprehensive study of the optical properties of both neutral and oxidized oligothienoacenes is needed.

In this contribution, we report for the first time on the physicochemical characterization of a whole series of fully fused oligothiophenes, ranging in length from the tetramer to the octamer (Figure 1), by means of a variety of experimental and theoretical tools, with the aim of establishing precise structure-property relationships. The optical properties of these triisopropylsilyl end-capped oligothienoacenes (TIPS-Tn-TIPS, in which $n$ indicates the number of fused thiophene rings arranged in an all-anti configuration) are studied by means of UV/Vis absorption and fluorescence emission spectroscopies, both in solution at room temperature and in solid glassy matrices of 2-methyltetrahydrofuran (2-MeTHF) at $80 \mathrm{~K}$. Well-resolved vibronic progressions are observed at low temperature, both in passing from the ground to the first excited singlet state and vice versa. The oxidized species are studied by means of in situ UV/Vis/ NIR spectroelectrochemistry. The three shortest members of the family give rise to stable radical cations, whereas the 
heptamer and octamer also give rise to stable dications. The presence of bulky TIPS groups at the terminal $\alpha$-positions of the oligothienyl backbone precludes the known tendency of common oligothiophenes towards the $\pi$-dimerization of the corresponding radical cations. Thus, this investigation provides a unique opportunity to characterize fully the variety of doped species along a homologous series of oligothienoacenes in the absence of disturbing processes such as $\pi$-dimerization. The experimental results are interpreted with the help of density functional theory (DFT) and time-dependent DFT (TDDFT) quantum-chemical calculations performed at the B3LYP/6-31G** level. Minimum-energy molecular structures, as well as one-electron vertical excitations, are computed for both neutral and oxidized molecules.

\section{Results and Discussion}

Electrochemical and structural properties: To gain an insight into the behavior of the TIPS-T $\boldsymbol{n}$-TIPS family against oxidation, their electrochemical and structural properties were studied by cyclic voltammetry and DFT//B3LYP/6-31G** calculations, respectively. The cyclic voltammograms are shown in Figure 2 along with the oxidation potentials $E_{\mathrm{ox}}^{\mathrm{o}}$. The values of $E_{\mathrm{ox}}^{\mathrm{o}}$ are determined from the center voltage between the peak for the oxidation of the sample and the peak for the reduction of the oxidized sample. Figure 3 displays the evolution of the first oxidation potentials, as well as the computed adiabatic ionization potentials with the reciprocal number of thiophene units $(1 / n)$.

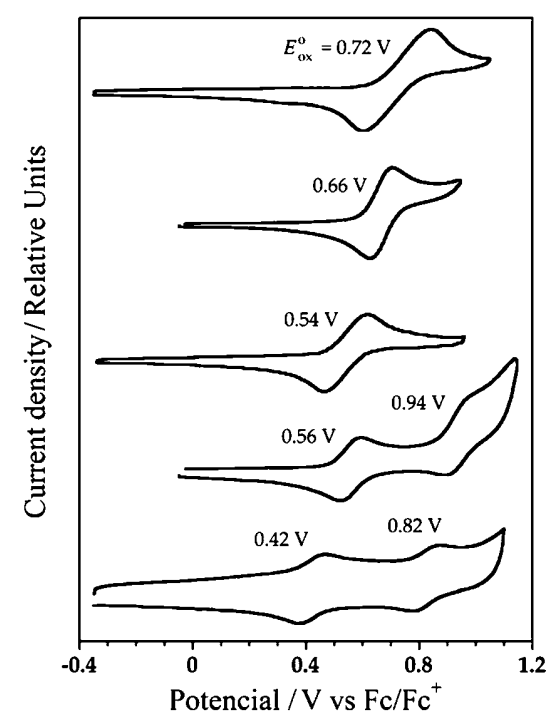

Figure 2. Cyclic voltammograms of TIPS-Tn-TIPS ( $n=4-8$; from top to bottom). The values of the oxidation potentials $E^{\circ}$ ox , referred to the $\mathrm{Fc}$ / $\mathrm{Fc}^{+}$couple, are given. Measurement conditions: a) even oligomers $(n=4$, 6, and 8): $\mathrm{Bu}_{4} \mathrm{NPF}_{6}(0.1 \mathrm{M})$ in $o$-dichlorobenzene; scan rate $50 \mathrm{mV} \mathrm{s}^{-1}$, b) odd oligomers $(n=5$ and 7$): \mathrm{Bu}_{4} \mathrm{NClO}_{4}(0.1 \mathrm{M})$ in dichloromethane; scan rate $100 \mathrm{mVs}^{-1}$.
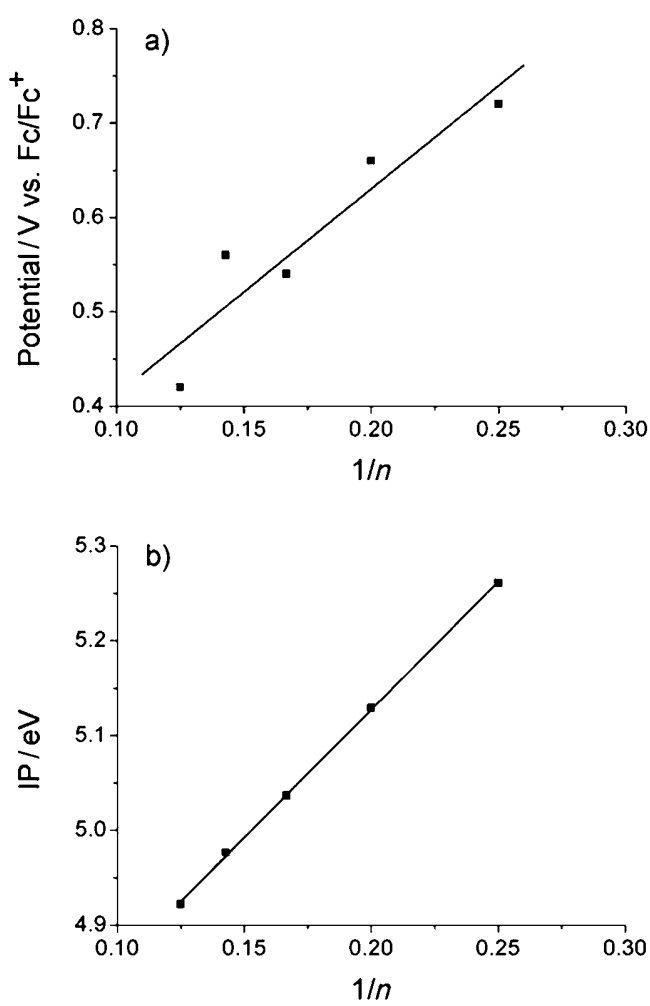

Figure 3. Dependence of a) the first oxidation potential, and b) the theoretically calculated adiabatic ionization potential (IP) on the reciprocal number of thiophene units $(1 / n)$ for TIPS-Tn-TIPS.

All the TIPS-Tn-TIPS oligothienoacenes show reversible oxidation waves (see Figure 2) indicative of the significant stability of the produced cationic species in these rigid ladder skeletons. Whereas the tetramer, pentamer, and hexamer present a unique oxidation process, the heptamer and octamer exhibit a second oxidation wave, showing that the extension of the $\pi$-conjugated backbone up to seven or more fused thiophene rings allows the formation of the dication. The first oxidation potential decreases as the number of thiophene rings is increased (see Figure $3 \mathrm{a}$ ). Deviations from linearity are clearly due to the slightly different sets of measurements performed for odd and even oligomers (all versus $\mathrm{Fc} / \mathrm{Fc}^{+}$, but in different solvents). The second electrochemical potential also decreases in passing from the heptamer $(0.94 \mathrm{~V})$ to the octamer $(0.82 \mathrm{~V})$. Compared to nonfused $\alpha$-oligothiophenes, all-anti oligothienoacenes present similar oxidation potentials. For instance, the first oxidation potentials measured for TIPS-T5-TIPS and TIPS-T7-TIPS are almost identical to those reported for $\alpha$-terthiophene and $\alpha$-quaterthiophene. ${ }^{[39]}$ Hence, the extra sulfur atoms incorporated by oligothienoacenes do not appear to be particularly influential for the oxidation energy.

Figure $3 \mathrm{~b}$ shows the adiabatic ionization potentials (IPs) computed for TIPS-Tn-TIPS at the B3LYP/6-31G** level in $o$-dichlorobenzene. IPs were calculated as the energy difference between the minimum-energy optimized structures of the radical cation and the neutral molecule. The IP value decreases with oligomer length, due to the extension of the 
$\pi$-conjugated backbone, and passes from $5.26 \mathrm{eV}$ for TIPST4-TIPS to $4.92 \mathrm{eV}$ for TIPS-T8-TIPS. This decrease of $0.34 \mathrm{eV}$ is comparable to the diminution of $0.30 \mathrm{~V}$ measured for the first oxidation potential. Indeed, the calculated IPs display an almost perfect linear dependence on the reciprocal number of thiophene units $(1 / n)$, with the line running parallel to the straight line fitted to the experimental redox potentials (see Figure $3 \mathrm{a}$ and $3 \mathrm{~b}$ ).

To investigate the effect of oxidation on the molecular structure of TIPS-Tn-TIPS oligothienoacenes, geometry optimizations of neutral, radical cation, and dication species were performed at the B3LYP/6-31G** level. Table 1 sum-

Table 1. B3LYP/6-31G** optimized bond lengths $[\AA]$ calculated for TIPS-T7-TIPS in its neutral, radical cation, and dication states. X-ray data from ref. [5] are included for the neutral molecule for comparison.

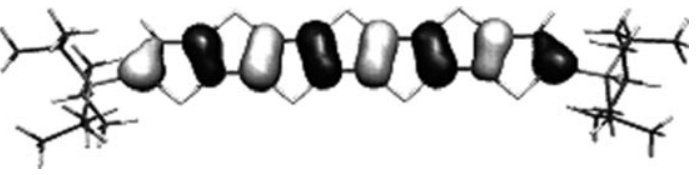

\begin{tabular}{lllll}
\hline Bond number & X-ray & Neutral & Cation & Dication \\
\hline $\mathrm{C}-\mathrm{C}$ & & & & \\
1 & 1.375 & 1.380 & 1.390 & 1.408 \\
2 & 1.417 & 1.420 & 1.407 & 1.391 \\
3 & 1.385 & 1.394 & 1.411 & 1.430 \\
4 & 1.420 & 1.419 & 1.398 & 1.378 \\
5 & 1.389 & 1.396 & 1.421 & 1.445 \\
6 & 1.412 & 1.417 & 1.392 & 1.373 \\
7 & 1.387 & 1.397 & 1.423 & 1.446 \\
8 & 1.416 & 1.417 & 1.390 & 1.371 \\
$\mathrm{C}-\mathrm{S}$ & & & & \\
9 & 1.749 & 1.772 & 1.762 & 1.747 \\
10 & 1.721 & 1.737 & 1.740 & 1.749 \\
11 & 1.740 & 1.762 & 1.761 & 1.760 \\
12 & 1.733 & 1.756 & 1.757 & 1.759 \\
13 & 1.734 & 1.756 & 1.755 & 1.756 \\
14 & 1.727 & 1.756 & 1.757 & 1.761 \\
15 & 1.741 & 1.756 & 1.757 & 1.759 \\
\hline
\end{tabular}

marizes the optimized bond lengths computed for TIPS-T7TIPS as a representative example, because this compound forms cation and dication species. At the top of Table 1, the topology of the highest occupied molecular orbital (HOMO) of TIPS-T7-TIPS is shown, to help in the interpretation of the structural changes the oligomer undergoes upon oxidation. The degree of aromatization/quinoidization of the conjugated backbone can be quantified easily by using the $\mathrm{C}-\mathrm{C}$ single/double bond length alternation (BLA) parameter. ${ }^{[40]}$ The BLA parameter is calculated for each thiophene ring as the difference between the length of the $\mathrm{C}_{\beta}-\mathrm{C}_{\beta}$ bond and the average of the two $\mathrm{C}_{\alpha}-\mathrm{C}_{\beta}$ bonds. An aromatic ring is thus characterized by a positive BLA value, while a quinoid ring shows a negative BLA value. The BLA values calculated for neutral TIPS-T7-TIPS remain almost constant along the chain, slightly diminishing from outer to inner rings $(0.033,0.024,0.022,0.021,0.022,0.024,0.033 \AA)$. The outer rings present a higher BLA value $(0.033 \AA)$ due to terminal effects. The BLA values are significantly smaller than those computed for $\alpha$-quaterthiophene, a nonfused oligomer with the same number of double $\mathrm{C}=\mathrm{C}$ bonds, for which BLA values of 0.033 and $0.050 \AA$ are obtained for inner and outer rings, respectively, at the B3LYP/6-31G** level. The equalization of the $\mathrm{C}-\mathrm{C}$ bonds in TIPS-T7-TIPS has to be attributed to the rigidity and fused nature of the backbone in oligothienoacenes, and is in good agreement with experimental X-ray data (see Table 1 ) ${ }^{[5]}$ It is also to be stressed that the $\mathrm{C}-\mathrm{C}$ bond lengths in the TIPS-Tn-TIPS series present a small dependence on oligomer size (see Table S1 in the Supporting Information).

The oxidation process involves the extraction of electrons from the HOMO, thus weakening the bonding and antibonding interactions localized on $\mathrm{C}-\mathrm{C}$ double and single formal bonds, respectively. As a consequence, $\mathrm{C}=\mathrm{C}$ double bonds lengthen, and $\mathrm{C}-\mathrm{C}$ single bonds shorten, and a reversal of the single/double bond pattern (quinoidization) takes place upon oxidation. For instance, bond number 7 in Table 1 undergoes a lengthening from $1.397 \AA$ in the neutral state to 1.423 and $1.446 \AA$ in the cation and dication states, respectively. In contrast, bond number 8 (the central bond, see Figure 1) exhibits a shortening from $1.417 \AA$ in the neutral molecule to 1.390 and $1.371 \AA$ in the two charged species, respectively. The BLA values computed for the cation $(0.017,-0.018,-0.030,-0.033,-0.030,-0.018,0.017 \AA)$ and the dication $(-0.028,-0.060,-0.073,-0.076,-0.073$, $-0.060,-0.028 \AA$ ) clearly illustrate the quinoidization of the conjugated carbon skeleton. The degree of quinoidization of the oligomer becomes more accentuated in passing from outer to inner thiophene rings, and a fully quinoid structure is found for the dication. $\mathrm{C}-\mathrm{S}$ bond lengths reach a rapid saturation in going from outer to inner rings in neutral TIPS-Tn-TIPS (see Table 1 for TIPS-T7-TIPS). Upon oxidation they remain almost constant, due to the fact that sulfur atoms hardly participate in the HOMO.

Figure 4 shows the NPA charge distribution calculated for TIPS-T7-TIPS in neutral, radical cation, and dication states. The oligothienyl backbone and the triisopropyl groups concentrate the electronic density and present negative net charges. The silicon atoms compensate those charges by ac-

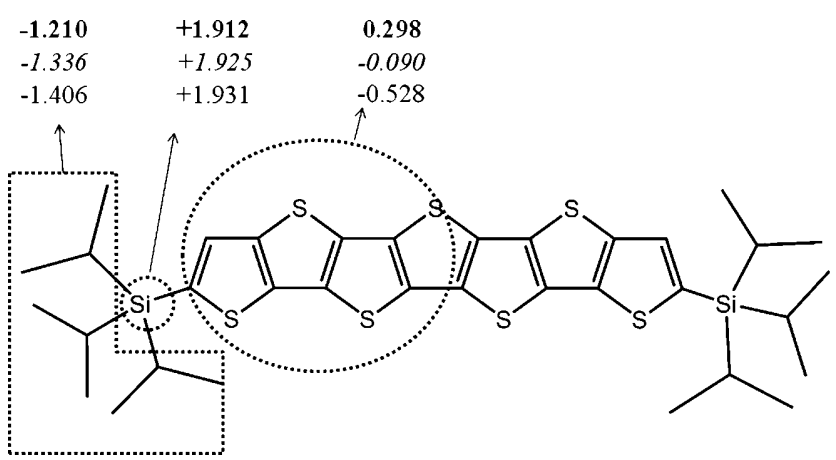

Figure 4. B3LYP/6-31G**-NPA c-harges calculated for TIPS-T7-TIPS in neutral (plain text), radical cation (italics), and dication (bold) states. TIPS-T7-TIPS has been divided into three parts (dotted lines): 1) half of oligothienyl backbone, 2) silicon atom, and 3) triisopropyl group. 
cumulating a positive charge of +1.931 due to their high electropositive character. Upon oxidation, electronic density is mainly extracted from the oligothienyl backbone, which reduces its charge from -1.056 in the neutral molecule to -0.180 in the cation, and to 0.596 in the dication. This implies the extraction of $85 \%$ (neutral $\rightarrow$ cation) and $74 \%$ (cation $\rightarrow$ dication) of the electron density from the oligothienyl backbone. At the same time, the positive charge borne by the silicon atoms remains almost unaffected, and the negative charge concentrated by the triisopropyl groups decreases slightly (neutral: -1.406 ; cation: -1.336 ; dication: -1.210). Therefore, electrons are mainly taken from the oligothienoacene skeleton, in agreement with the larger changes calculated for the bonds forming the conjugated fused skeleton.

Optical properties of neutral
TIPS-T $n$-TIPS oligothienoacenes: Figure 5 displays the normalized UV/Vis absorption spectra of TIPS-Tn-TIPS, as well as the fluorescence spectra of TIPS-T5-TIPS and TIPS-T8TIPS, recorded in MeTHF both at room and at low temperature. Table 2 shows the maximum absorption and fluorescence wavelengths of the various peaks forming the vibronically structured absorption and emission bands recorded at room temperature. The TIPSTn-TIPS compounds show a strong electromagnetic absorption in the near-UV/visible range, with the longest absorption maximum around 350$430 \mathrm{~nm}$, and a second weak band at higher energies.

At room temperature, wellresolved vibronic structures are evident in the UV/Vis absorption spectra of all the TIPS-TnTIPS oligomers. The appearance of a vibronic structure in solution is a fairly unique property of oligothienoacenes, and is generally not observed for nonfused $\alpha$-oligothiophenes. ${ }^{[41]}$ The broad structureless absorption bands commonly observed for $\alpha$-oligothiophenes are due to inhomogeneous broadening produced by the conformational disorder result-
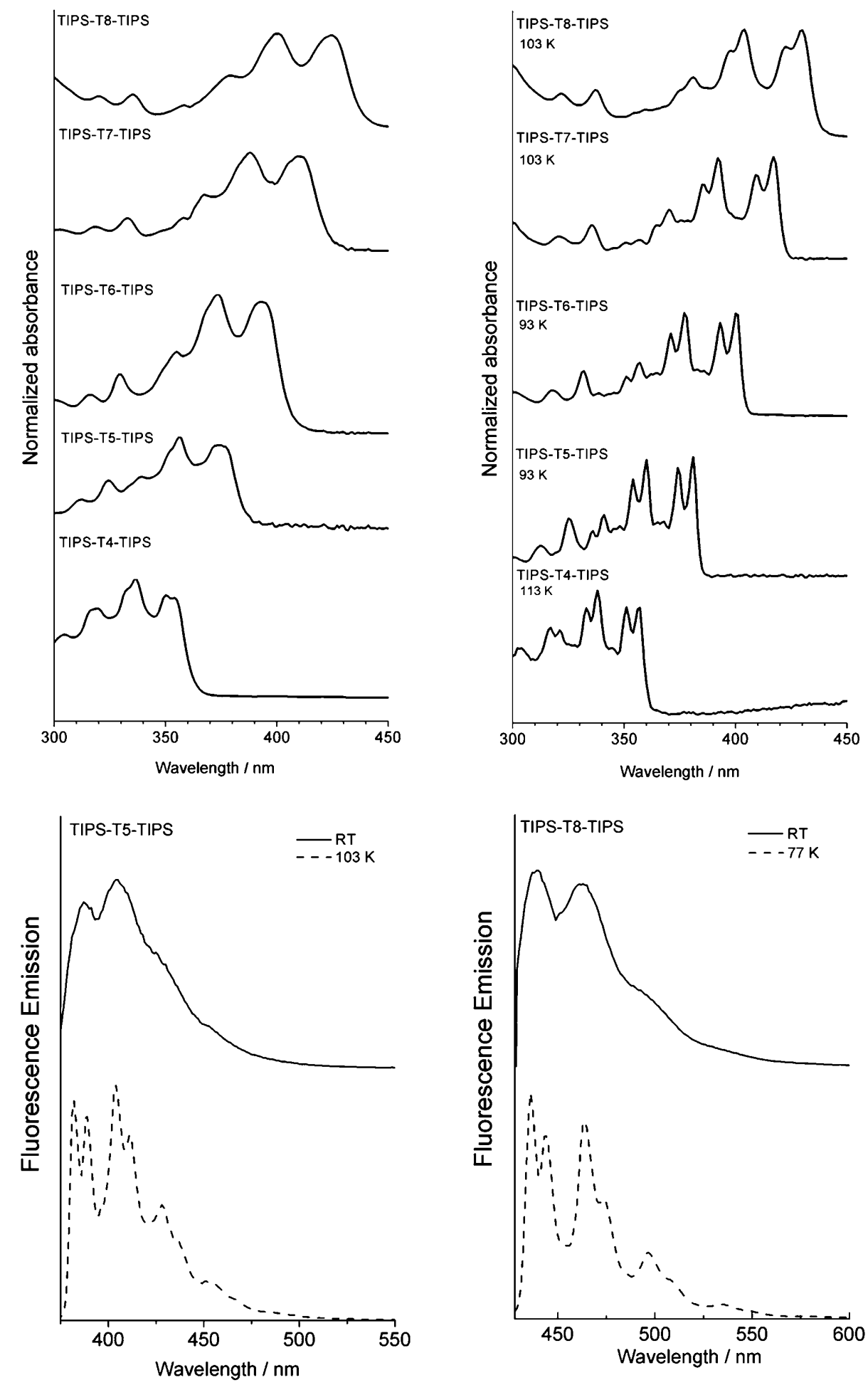

Figure 5. Normalized UV/Vis absorption spectra of TIPS-Tn-TIPS oligothienoacenes in MeTHF at room temperature (top left) and at low temperature (top right). Normalized fluorescence emission spectra for TIPS-T5TIPS (bottom left) and TIPS-T8-TIPS (bottom right) in MeTHF at room and low temperature. 
Table 2. UV/Vis absorption and fluorescence emission maxima observed for TIPS-Tn-TIPS at room temperature.

\begin{tabular}{lll}
\hline Compound & $\lambda_{\max }$ absorption $[\mathrm{nm}]$ & $\lambda_{\max }$ emission $[\mathrm{nm}]$ \\
\hline TIPS-T4-TIPS & $350,337,319,305$ & \\
TIPS-T5-TIPS & $375,356,339,324,312$ & $387,404,428$ \\
TIPS-T6-TIPS & $393,373,355,329,316$ & \\
TIPS-T7-TIPS & $409,388,367,358,333,318$ & \\
TIPS-T8-TIPS & $425,400,379,335,320$ & $440,463,500$ \\
\hline
\end{tabular}

ing from the internal rotation between thiophene rings. Upon decreasing the temperature, the degree of conformational disorder is reduced and the absorption bands display well-resolved vibronic structures. ${ }^{[41]}$ The reason for the vibronic resolution of the absorption bands in the room-temperature spectra of TIPS-Tn-TIPS oligothienoacenes is the rigidity of the fully fused thiophene backbone, which drastically reduces the conformational disorder and, therefore, the inhomogeneous broadening. The fluorescence emission spectra recorded at room temperature (see Figure 5) also show well-resolved vibronic structures.

The partially resolved vibronic structures observed at room temperature in the UV/Vis absorption spectra of TIPS-Tn-TIPS undergo a large transformation with temperature. Upon cooling, the vibronic peaks sharpen and become more intense. The lowest energy optical absorption clearly shows two different vibronic progressions with different energy splittings, for which up to three replicas are seen. The vibronic progressions correspond to vibrational modes with energies of $v_{1} \approx 180 \mathrm{meV} \quad\left(\approx 1525 \mathrm{~cm}^{-1}\right)$ and $v_{2}$ $\approx 56 \mathrm{meV}\left(\approx 480 \mathrm{~cm}^{-1}\right)$. The first two peaks of both progressions have similar intensities. Despite the major changes observed in the spectra, the absorption onset remains unchanged with respect to the room-temperature spectra. The higher resolution obtained for the low-temperature spectra (see Figure 5, top) can be interpreted as due to the loss of some remaining conformational disorder associated with low-energy, out-of-plane molecular vibrations. This disorder seems to persist to some extent even at low temperatures for the longer oligomers, as the resolution of the spectra diminishes with oligomer size (see Figure 5, top right).

The temperature lowering also has a strong effect on the fluorescence of oligothienoacenes. The fluorescence spectra recorded in MeTHF at $103 \mathrm{~K}$ for TIPS-T5-TIPS and at $77 \mathrm{~K}$ for TIPS-T8-TIPS (Figure 5, bottom) also exhibit two resolved vibronic progressions with energy splittings $(\approx 1400$ and $\approx 450 \mathrm{~cm}^{-1}$ ) smaller than those observed for the absorption spectra $(\approx 1525$ and $\left.\approx 480 \mathrm{~cm}^{-1}\right)$. These progressions can be assigned on the basis of the vibrational properties calculated for TIPS-Tn-TIPS compounds in their ground state. ${ }^{[42]}$ The first progression can be atrespectively.
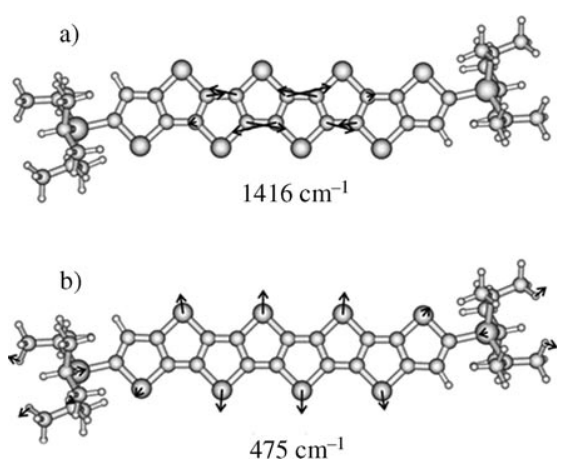

Figure 6. Selected B3LYP/6-31G** vibrational eigenvectors calculated for TIPS-T8-TIPS in its ground state. Theoretical wavenumbers are scaled down by a factor of $0.96 .^{[43]}$

tributed to totally symmetric, collective $v(\mathrm{C}-\mathrm{C})$ stretching modes of the oligothienoacene core, as depicted in Figure 6 a for TIPS-T8-TIPS. The second progression could be the result of in-plane bending modes of the thiophene rings, such as that displayed in Figure $6 \mathrm{~b}$. These two modes are calculated at 1416 and $475 \mathrm{~cm}^{-1}$, respectively, for TIPS-T8TIPS, in good agreement with the splittings observed in the fluorescence spectra.

Highly resolved fluorescence spectra have previously been observed for nonfused oligothiophenes such as bithiophene, terthiophene, and quaterthiophene in $n$-alkanes at $4.2 \mathrm{~K},{ }^{[44-47]}$ but for these oligomers the $v_{2} \approx 480 \mathrm{~cm}^{-1}$ progression was not observed. The observation of two different progressions has also been reported for oligoethylenedioxythiophenes (OEDOTs) by Wasserberg et al. ${ }^{[15]}$ These oligomers present partially rigid molecular structures due to the noncovalent interactions between sulfur and oxygen atoms in adjacent EDOT units.

To provide insight into the nature of the UV/Vis absorptions experimentally observed for TIPS-Tn-TIPS, the singlet excited electronic states of all these oligomers were calculated at the B3LYP/6-31G** level using the TDDFT approach and the optimized ground-state molecular geometries. Vertical excitation energies and oscillator strengths calculated both in the gas phase and in THF are listed in Table 3, along with the description of the electronic transitions in terms of

Table 3. TDDFT//B3LYP/6-31G** vertical one-electron excitations [in nm] calculated for TIPS-Tn-TIPS related to the strongest UV/Vis absorptions observed experimentally.

\begin{tabular}{lllll}
\hline Compound & Expt $^{[\mathrm{a}]}$ & TDDFT $(f)^{[\mathrm{b}, \mathrm{c}]}$ & ${\text { TDDFT }(f)^{[\mathrm{c}, \mathrm{d}]}}$ & Description $^{[\mathrm{e}]}$ \\
\hline TIPS-T4-TIPS & $350(337,319)$ & $346(1.12)$ & $354(1.26)$ & $\mathrm{H} \rightarrow \mathrm{L}$ \\
& 305 & $307(0.07)$ & $306(0.12)$ & $\mathrm{H}-1 \rightarrow \mathrm{L}$ \\
TIPS-T5-TIPS & $375(356,339)$ & $372(1.32)$ & $382(1.47)$ & $\mathrm{H} \rightarrow \mathrm{L}$ \\
& $324(312)$ & $316(0.08)$ & $317(0.14)$ & $\mathrm{H}-1 \rightarrow \mathrm{L}$ \\
TIPS-T6-TIPS & $393(373,355)$ & $395(1.57)$ & $407(1.73)$ & $\mathrm{H} \rightarrow \mathrm{L}$ \\
& $329(316)$ & $324(0.08)$ & $325(0.14)$ & $\mathrm{H}-1 \rightarrow \mathrm{L}$ \\
TIPS-T7-TIPS & $409(388,367,358)$ & $416(1.78)$ & $430(1.94)$ & $\mathrm{H} \rightarrow \mathrm{L}$ \\
& $333(318)$ & $330(0.06)$ & $332(0.12)$ & $\mathrm{H}-1 \rightarrow \mathrm{L}$ \\
TIPS-T8-TIPS & $425(400,379)$ & $435(2.02)$ & $450(2.18)$ & $\mathrm{H} \rightarrow \mathrm{L}$ \\
& $335(320)$ & $334(0.09)$ & $336(0.16)$ & $\mathrm{H}-2 \rightarrow \mathrm{L}$ \\
\hline
\end{tabular}

[a] Values within parentheses correspond to vibronic peaks. [b] Calculated in gas phase. [c] Calculated in THF. [d] Oscillator strength $(f)$ values are given in parentheses. [e] HOMO and LUMO are abbreviated as H and L, 
the dominant one-electron excitations. Figure 7 depicts the topologies of selected MOs for TIPS-T7-TIPS as a guide to the TDDFT description of the excited states and optical absorptions.

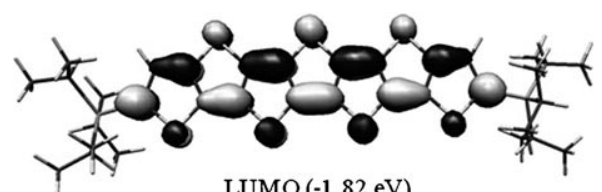

$\operatorname{LUMO}(-1.82 \mathrm{eV})$

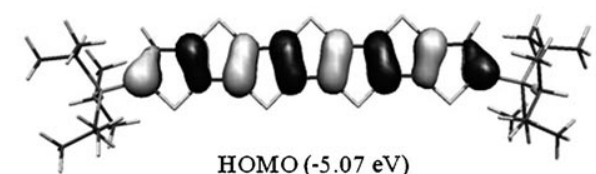

$\operatorname{HOMO}(-5.07 \mathrm{eV})$

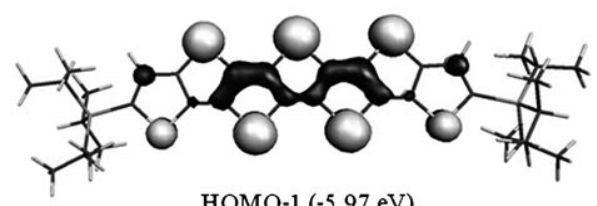

HOMO-1 (-5.97 eV)

Figure 7. B3LYP/6-31G** electronic density contours $\left(0.03 \mathrm{ebohr}^{-3}\right)$ and energies calculated for selected MOs of TIPS-T7-TIPS.

TDDFT calculations predict the existence of a very strong electronic transition in the near-UV/visible range, followed by a weaker electronic transition at higher energies $(\approx 320 \mathrm{~nm})$ for all the TIPS-Tn-TIPS oligothienoacenes (see Table 3). The first transition, computed at $346 \mathrm{~nm}(f=1.12)$ for TIPS-T4-TIPS, $372 \mathrm{~nm}(f=1.32)$ for TIPS-T5-TIPS, $395 \mathrm{~nm}(f=1.57)$ for TIPS-T6-TIPS, $416 \mathrm{~nm}(f=1.78)$ for TIPS-T7-TIPS, and $435 \mathrm{~nm}(f=2.02)$ for TIPS-T8-TIPS, corresponds to the excitation to the first singlet excited state $\left(\mathrm{S}_{0} \rightarrow \mathrm{S}_{1}\right)$, and shows an increase in intensity as the oligomer lengthens. The $S_{0} \rightarrow S_{1}$ electronic transition is mainly described by the one-electron excitation from the HOMO to the lowest unoccupied molecular orbital (LUMO), which are mainly delocalized on the conjugated carbon skeleton (see Figure 7). The calculated excitation energies are very close to the first absorption maxima measured experimentally, with the effect of the solvent on the computed energies being quite small (Table 3 ). The plot of the $S_{0} \rightarrow S_{1}$ excitation energy versus the reciprocal number of fused thiophene rings (Figure 8) shows a linear correlation in good agreement with the experimental data. The $\mathrm{S}_{0} \rightarrow \mathrm{S}_{1}$ transition undergoes a large redshift with the length of the oligomer, which can be taken as proof of the existence of a remarkable $\pi$ conjugation along the fully fused thiophene backbone.

TDDFT//B3LYP/6-31G** calculations also predict the appearance of a second absorption band quite close in energy to the HOMO $\rightarrow$ LUMO transition (see Table 3 ). This electronic transition is much weaker $(f<0.10)$ and is computed in the 305-335 nm range in good agreement with the experimental absorption band observed in this region. The transition corresponds to the $S_{0} \rightarrow S_{2}$ excitation $\left(S_{0} \rightarrow S_{3}\right.$ for TIPS-

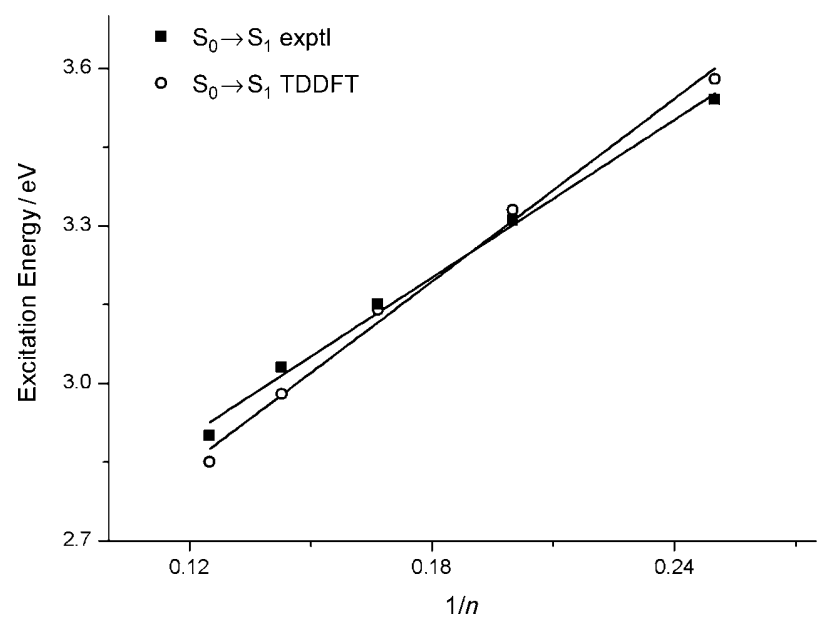

Figure 8. Experimental and theoretical (TDDFT//B3LYP/6-31G**) energies of the lowest electronic transition $\mathrm{S}_{0} \rightarrow \mathrm{S}_{1}$ of TIPS-Tn-TIPS as a function of the reciprocal number of thiophene rings $(1 / n)$ in the oligomer.

T8-TIPS), and is described by the one-electron promotion from the HOMO-1 to the LUMO (HOMO-2 $\rightarrow$ LUMO for TIPS-T8-TIPS). The topology of the HOMO-2 in TIPST8-TIPS is indeed the same as the topology of the HOMO-1 in the shorter oligomers. The electronic transition involves some electron transfer from sulfur atoms, mainly contributing to the $\mathrm{HOMO}-1$, to the carbon skeleton, in which the LUMO mostly resides (see Figure 7). TDDFT calculations therefore allow a quantitative description of the absorption spectra of TIPS-Tn-TIPS. The absorption bands are assigned to the $\mathrm{S}_{0} \rightarrow \mathrm{S}_{1}$ and $\mathrm{S}_{0} \rightarrow \mathrm{S}_{2}\left(\mathrm{~S}_{0} \rightarrow \mathrm{S}_{3}\right.$ for TIPS-T8-TIPS) electronic excitations, the first one being responsible for the highly resolved vibronic structure observed experimentally.

Optical properties of oxidized TIPS-T $n$-TIPS oligothienoacenes: To investigate the optical properties of oxidized TIPS-Tn-TIPS, in situ UV/Vis/NIR spectroelectrochemistry experiments were performed. Figure 9 displays the evolution of the spectra of TIPS-Tn-TIPS $(n=5-8)$ in $o$-dichlorobenzene solution during electrochemical oxidation in tetrabutylammonium perchlorate $\left(n \mathrm{Bu}_{4} \mathrm{NClO}_{4}\right)$. Table 4 summarizes the absorption features observed experimentally, together with those calculated at the B3LYP/6-31G** level for the oxidized species. Figure 10 schematizes the change the monoelectronic levels around the HOMO-LUMO gap undergo upon the oxidation process.

Figure 9 displays the spectra of neutral compounds registered at $0 \mathrm{~s}$, which were discussed in the previous section. At short recording times (10 and $50 \mathrm{~s}$ ), two new absorption bands appear, at 510 and $842 \mathrm{~nm}$ for TIPS-T5-TIPS, 544 and $943 \mathrm{~nm}$ for TIPS-T6-TIPS, 575 and $1028 \mathrm{~nm}$ for TIPS-T7TIPS, and 605 and $>1100 \mathrm{~nm}$ (detection limit of the instrument) for TIPS-T8-TIPS, which correspond to the formation of the radical cation [TIPS-Tn-TIPS] ${ }^{*+}$. The high-energy band is accompanied by a vibronic transition on its highenergy side $(470,498,523$, and $540 \mathrm{~nm}$ for $n=5-8$, respec- 

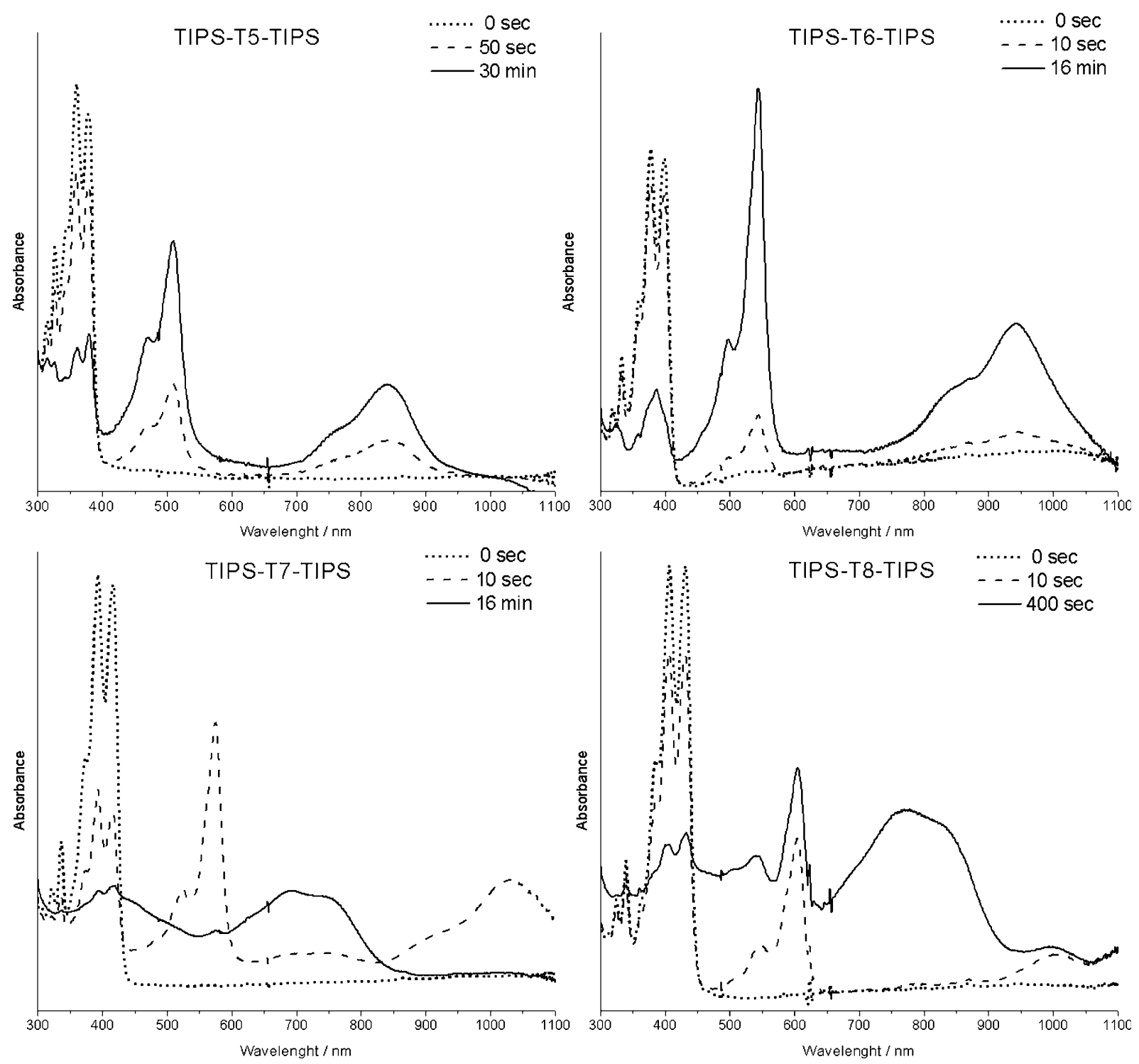

Figure 9. UV/Vis/NIR spectra recorded in $o$-dichlorobenzene solution during the electrochemical oxidation of TIPS-Tn-TIPS compounds. The voltage applied vs. $\mathrm{Ag} / \mathrm{Ag}^{+}$was $0.9 \mathrm{~V}$ for $n=5$ and 6 , and $1.2 \mathrm{~V}$ for $n=7$ and 8 .

tively). The low-energy band in the $850-1100 \mathrm{~nm}$ range is very broad and has a less defined vibronic structure. The

Table 4. Experimental and calculated absorption wavelengths [in $\mathrm{nm}$ ] of TIPS-Tn-TIPS $(n=5-8)$ cations and dications.

\begin{tabular}{|c|c|c|c|c|c|c|}
\hline \multirow[b]{2}{*}{ Compound } & \multicolumn{3}{|c|}{ TIPS-Tn-TIPS ${ }^{+}$} & \multicolumn{3}{|c|}{ TIPS-Tn-TIPS $^{2+}$} \\
\hline & $\operatorname{Exptl}^{[\mathrm{a}]}$ & $\operatorname{TDDFT}(f)^{[\mathrm{b}, \mathrm{c}]}$ & Description $^{[\mathrm{d}]}$ & Exptl $^{[\mathrm{a}]}$ & TDDFT $(f)^{[\mathrm{b}, \mathrm{c}]}$ & Description $^{[\mathrm{d}]}$ \\
\hline \multirow[t]{2}{*}{ TIPS-T5-TIPS } & 842 & $768(0.46)$ & $\mathrm{H} \rightarrow \mathrm{S}$ & & & \\
\hline & $510(470)$ & $463(1.02)$ & $\mathrm{S} \rightarrow \mathrm{L}$ & & & \\
\hline \multirow[t]{2}{*}{ TIPS-T6-TIPS } & 943 & $867(0.42)$ & $\mathrm{H} \rightarrow \mathrm{S}$ & & & \\
\hline & $544(498)$ & $537(0.46)$ & $\mathrm{S} \rightarrow \mathrm{L}$ & & & \\
\hline \multirow[t]{2}{*}{ TIPS-T7-TIPS } & 1028 & $929(0.60)$ & $\mathrm{H} \rightarrow \mathrm{S}$ & $749(691)$ & $754(1.82)$ & $\mathrm{H} \rightarrow \mathrm{L}$ \\
\hline & $575(523)$ & $548(1.16)$ & $\mathrm{S} \rightarrow \mathrm{L}$ & & & \\
\hline \multirow[t]{2}{*}{ TIPS-T8-TIPS } & $>1100$ & $1031(0.80)$ & $\mathrm{H} \rightarrow \mathrm{S}$ & $830(769)$ & $763(2.24)$ & $\mathrm{H} \rightarrow \mathrm{L}$ \\
\hline & $605(540)$ & $569(1.54)$ & $\mathrm{S} \rightarrow \mathrm{L}$ & & & \\
\hline
\end{tabular}

[a] Recorded in $o$-dichlorobenzene. Values within parentheses correspond to vibronic peaks. [b] Calculated in chlorobenzene solution. [c] Oscillator strength $(f)$ values are given in parentheses. [d] HOMO, SOMO, and LUMO are abbreviated as $\mathrm{H}, \mathrm{S}$, and L, respectively. isosbestic point around $400-450 \mathrm{~nm}$ indicates the interconversion of two species, consistent with the oxidation of

TIPS-Tn-TIPS into [TIPS-TnTIPS] ${ }^{\cdot+}$. The appearance of two low-energy spectral features associated with the formation of the radical cation is in agreement with the numerous spectroelectrochemical ${ }^{[19-31]}$ and quantum-chemical ${ }^{[32-38]}$ studies reported for a great variety of $\alpha$-oligothiophenes. The in situ UV/Vis/NIR spectra were recorded at room temperature and for low TIPS-Tn-TIPS concentrations, and, therefore, show no indication of the for- 


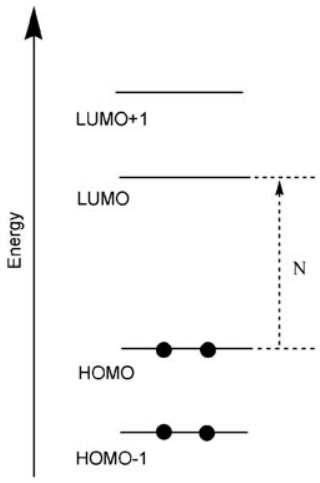

Neutral compound

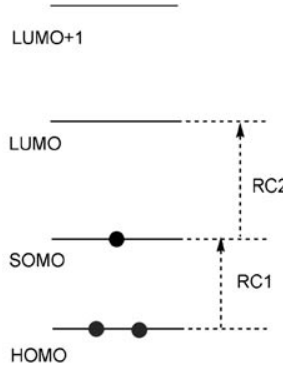

Radical Cation
LUMO+2

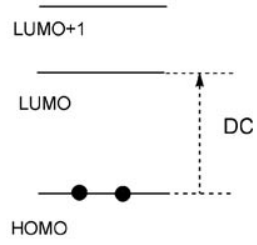

Dication
Figure 10. Schematic representation of the frontier monoelectronic levels and the dipole-allowed transitions of TIPS-T $n$-TIPS in neutral $(\mathrm{N})$, radical cation (RC), and dication (DC) oxidation states.

mation of cation radical dimers. The formation of dimers is furthermore hindered by the bulky TIPS groups incorporated in these oligomers in their terminal positions.

Upon further oxidation, only the longer oligothienoacenes are able to form dication species, in agreement with the observations from the cyclic voltammetry study. As Figure 9 highlights, only the radical cations of the heptamer and the octamer are converted to the [TIPS-T7-TIPS $]^{2+}$ and [TIPST8-TIPS $]^{2+}$ dications. The dications are characterized by a new broad absorption in the $750-850 \mathrm{~nm}$ range, and the disappearance of the bands of the cation at lower and higher energies. The wavelengths recorded are similar to those previously reported for end-capped $\alpha$-quaterthiophene and $\alpha$ pentathiophene, ${ }^{[25]}$ which bear a number of conjugated $\mathrm{C}=\mathrm{C}$ double bonds ( 8 and 10 , respectively), comparable to TIPST7-TIPS (8) and TIPS-T8-TIPS (9).

Vertical electronic transitions for the radical cation and dication species of TIPS-Tn-TIPS were computed using the TDDFT approach and the PCM model to introduce solvent effects (Table 4). TDDFT calculations predict two intense electronic transitions for radical cations at lower energies than those computed for the neutral oligomers. For example, the energies calculated for these two transitions are $1.33 \mathrm{eV}$ $(929 \mathrm{~nm}, f=0.60)$ and $2.26 \mathrm{eV}(548 \mathrm{~nm}, f=1.16)$ for [TIPST7-TIPS $]^{\cdot+}$, slightly overestimating the experimental values observed at $1.21 \mathrm{eV}(1028 \mathrm{~nm})$ and $2.16 \mathrm{eV}(575 \mathrm{~nm})$. TDDFT calculations also reproduce the ratio between the experimental intensities and the steady redshift that both absorptions undergo with the extension of the chain length. As sketched in Figure 10, the new emerging transitions are mainly described by the $\mathrm{HOMO} \rightarrow$ SOMO (singly occupied molecular orbital) and the SOMO $\rightarrow$ LUMO one-electron excitations.

For the dication species, TDDFT calculations indicate the appearance of one strong electronic transition at intermediate energies with respect to the two transitions calculated for the radical cation (see Table 4). The transition results from the HOMO $\rightarrow$ LUMO excitation, in which the HOMO and the LUMO now correspond to the HOMO-1 and
HOMO of the neutral molecule (see Figure 10). The transition is calculated at $754 \mathrm{~nm}$ for [TIPS-T7-TIPS] ${ }^{2+}$, in good correlation with the experimental band measured at $749 \mathrm{~nm}$. TDDFT calculations are therefore shown to be very helpful in interpreting the evolution upon oxidation of the optical properties of the fully fused TIPS-Tn-TIPS compounds.

\section{Conclusion}

This work presents an analysis of the evolution of the structural, electrochemical, and optical properties within a complete family of triisopropylsilyl end-capped oligothienoacenes (TIPS-Tn-TIPS, $n=4-8$ ), which emerge as a potential class of fully fused $\pi$-conjugated compounds for organic electronics. The analysis is performed by combining cyclic voltammetry, a selection of spectroscopic tools (optical absorption, fluorescence emission, and in situ UV/Vis/NIR spectroelectrochemistry), and quantum-chemical calculations based on the density functional theory (DFT and TDDFT) approach.

Cyclic voltammetry reveals reversible redox behavior for all the TIPS-Tn-TIPS family, indicating the formation of stable radical cations for all these oligothienoacenes, and the formation of dications only for the heptamer and the octamer. Oxidation produces a reversal of the single/double $\mathrm{C}-\mathrm{C}$ bond pattern and leads to fully quinoid structures for the dications. The quinoidization of the molecular structure is more marked for the inner thiophene rings and decreases in going to the outer rings. DFT calculations predict that the electronic density is mainly extracted from the thienyl backbone. For TIPS-T7-TIPS, $0.85 \mathrm{e}$ and $0.74 \mathrm{e}$ are extracted from the backbone in the neutral $\rightarrow$ cation and cation $\rightarrow$ dication processes, respectively. The charges extracted are in good agreement with the changes predicted for the bond lengths upon oxidative doping, which are also localized in the fused conjugated backbone. The formation of stable cation and dication species and the delocalization of the charge on the conjugated chain make TIPS-Tn-TIPS compounds promising as hole-transporting materials for future applications in organic electronics.

The planarity and rigidity of fully fused TIPS-Tn-TIPS oligothienoacenes drastically affect their optical properties. Compared to their nonfused $\alpha$-oligothiophene partners, the absorption and fluorescence spectra of TIPS-Tn-TIPS show partially resolved vibronic structures even at room temperature, due to the absence of conformational disorder. Two well-resolved vibronic progressions are observed at low temperatures that can be associated with vibronic coupling with two different normal modes, with energies of $v_{1} \approx 180 \mathrm{meV}$ $\left(\approx 1525 \mathrm{~cm}^{-1}\right)$ and $v_{2} \approx 56 \mathrm{meV}\left(\approx 480 \mathrm{~cm}^{-1}\right)$. The progression due to the coupling with the lower frequency mode is not observed in nonfused oligothiophenes. TDDFT calculations have allowed a quantitative description of the absorption spectra, and the assignment of the electronic transitions responsible for the main observed bands. Both experimental and theoretical results find a remarkable bathochromic dis- 
persion of the electronic transitions as the chain length increases, which is indicative of the extension of the effective $\pi$-conjugation.

In situ UV/Vis/NIR spectroelectrochemistry in $o$-dichlorobenzene of TIPS-Tn-TIPS provides evidence of the formation of cation and dication species. Similarly to what is observed for other conjugated oligomers, two absorption bands emerge at low energies, corresponding to the formation of the radical cation in the first oxidation steps. The longest oligomers (heptamer and octamer) form the dication species, which is characterized by a unique absorption band at intermediate energies. The formation of cation radical dimers is not detected, probably due to the bulky triisopropylsilyl groups, which hinder noncovalent aggregation. TDDFT calculations taking into account solvent effects within the PCM model have helped in interpreting and assigning the optical data.

\section{Experimental Section}

The synthesis and purification of the TIPS-Tn-TIPS oligothienoacenes are described elsewhere. ${ }^{[5,14]} \mathrm{UV} / \mathrm{Vis} / \mathrm{NIR}$ absorption spectra were recorded at room temperature by means of an Agilent 8453 instrument equipped with a diode array for fast recording of all electromagnetic absorptions in the 190-1100 nm spectral region. Fluorescence emission spectra were measured using a JASCO FP-750 spectrofluorimeter. No fluorescent contaminants were detected upon excitation in the wavelength region of experimental interest. Solutions for emission measurements were prepared with an absorbance in the visible spectral region between 0.1 and 0.2. 2-Methyltetrahydrofuran (MeTHF) was pre-dried over $\mathrm{KOH}$ for three days, filtered, distilled from $\mathrm{CaH}_{2}$, and stored under an inert atmosphere. Sample solutions in MeTHF were prepared in a nitrogen-filled glove box at concentrations between $10^{-5}$ and $10^{-7} \mathrm{M}$. Lowtemperature spectra were recorded using an Oxford Optistat DN nitrogen bath cryostat with the capability to operate in the temperature range 77-500 K.

Electrochemical experiments were performed in $o$-dichlorobenzene (TIPS-T4-TIPS, TIPS-T6-TIPS, and TIPS-T8-TIPS) or dichloromethane (TIPS-T5-TIPS and TIPS-T7-TIPS) at $25^{\circ} \mathrm{C}$ under nitrogen in three-electrode cells. The supporting electrolyte was tetrabutylammonium hexafluorophosphate $\left(\mathrm{Bu}_{4} \mathrm{NPF}_{6}\right)$ or perchlorate $\left(\mathrm{Bu}_{4} \mathrm{NClO}_{4}\right)$, respectively. The counter electrode was platinum and the reference electrode was silver/ $0.1 \mathrm{~m}$ silver perchlorate in acetonitrile, but quoted potentials are always referred to the $\mathrm{Fc} / \mathrm{Fc}^{+}$couple. The working electrode was a platinum minidisc electrode $\left(0.003 \mathrm{~cm}^{2}\right)$. The voltammetric apparatus (AMEL, Italy) included a 551 potentiostat modulated by a 568 programmable function generator and coupled to a 731 digital integrator. UV/Vis/NIR absorption spectra during the electrochemical study of the compounds were recorded on a Perkin-Elmer Lambda 5 spectrometer. In situ UV/ Vis/NIR spectroelectrochemistry was performed by controlled potential electrolysis in an OTTLE cell equipped with a platinum minigrid working electrode and quartz optical windows.

DFT calculations were carried out by means of the Gaussian 03 program. ${ }^{[48]}$ All the calculations, including geometry optimizations of the ground state and electronic excitation energies, were performed with Becke's three-parameter B3LYP exchange-functional ${ }^{[49,50]}$ and the 6$31 \mathrm{G}^{* *}$ basis set. ${ }^{[51]}$ Oligothienoacenes with an even (odd) number of rings were optimized within $C_{i}\left(C_{2}\right)$ symmetry constraints. Radical cations were treated as open-shell systems and computed within the spin-unrestricted DFT (UDFT) approach, whereas dications were treated as closed-shell systems and calculated using the spin-restricted DFT approach.
Vertical electronic transition energies were computed at the B3LYP/6$31 \mathrm{G}^{* *}$ level using the TDDFT approach ${ }^{[52-54]}$ and the optimized groundstate molecular geometries. Solvent effects were considered within the SCRF (self-consistent reaction field) theory using the polarized continuum model (PCM) approach to model the interaction with the solvent. ${ }^{[55,56]}$ PCM calculations for the charged species were performed using the standard parameters implemented for chlorobenzene in Gaussian 03 and the dielectric constant of $o$-dichlorobenzene $(\varepsilon=10.12)$ to simulate the experimental recording conditions. TDDFT calculations for the neutral compounds were carried out both in the gas phase and in the presence of the solvent (THF). The differences found for the vertical excitation energies were smaller than $0.08 \mathrm{eV}$. Net atomic charges were calculated using the natural population analysis (NPA) included in the natural bond orbital (NBO) algorithm proposed by Weinhold and co-workers. ${ }^{[57-59]}$ Molecular orbitals were plotted using Molekel 4.3. ${ }^{[60]}$

\section{Acknowledgements}

Financial support from the Ministerio de Ciencia e Innovació (MICINN) of Spain (projects CTQ2006-14987-C02-01 and CTQ2006-14987-C02-02, CTQ2009-08790, CTQ2009-10098, and Consolider-Ingenio CSD200700010 in Molecular Nanoscience), the Junta de Andalucia (grant FQM0159, project P09-4708), the Generalitat Valenciana (grant ACOMP/ 2009/269), and European FEDER funds (project CTQ2006-14987-C02) are gratefully acknowledged. R.M.O. and J.A. are also grateful to the MICINN for personal doctoral grants.

[1] C. D. Dimitrakopoulos, P. Malefant, Adv. Mater. 2002, 14, 99.

[2] A. R. Murphy, J. M. J. Fréchet, Chem. Rev. 2007, 107, 1066.

[3] C. Goldmann, S. Hass, C. Krellner, K. P. Pernstich, D. J. Gundlanch, B. Batlogg, J. Appl. Phys. 2004, 96, 2080.

[4] R. M. Osuna, X. Zhang, A. J. Matzger, V. Hernández, J. T. López Navarrete, J. Phys. Chem. A 2006, 110, 5058

[5] X. Zhang, A. P. Côte, A. J. Matzger, J. Am. Chem. Soc. 2005, 127, 10502 .

[6] J.-L. Brédas, J. P. Calbert, D. A. Da Silva Filho, J. Cornil, Proc. Natl. Acad. Sci. USA 2002, 99, 5804.

[7] K. Xiao, Y. Liu, T. Qi, W. Zhang, F. Wang, J. Gao, W. Qiu, Y. Ma, G. Cui, S. Chen, X. Zhan, G. Yu, J. Qin, W. Hu, D. Zhu, J. Am Chem. Soc. 2005, 127, 13281.

[8] Y. Mazaki, K. Kobayashi, J. Chem. Soc. Perkin Trans. 2 1992, 761

[9] N. Sato, Y. Mazaki, K. Kobayashi, T. Kobayashi, J. Chem. Soc. Perkin Trans. 2 1992, 765.

[10] W. Schroth, E. Hintzsche, H. Viola, R. Winkler, H. Klose, R. Boese, R. Kempe, J. Sieler, Chem. Ber. 1994, 127, 401.

[11] W. Schroth, E. Hintzsche, M. Felicetti, R. Spitzner, J. Sieler, R. Kempe, Angew. Chem. 1994, 106, 808; Angew. Chem. Int. Ed. Engl. 1994, 33, 739 .

[12] W. Schroth, E. Hintzsche, H. Jordan, T. Jende, R. Spitzner, I. Thondorf, Tetrahedron 1997, 53, 7509.

[13] K. Oyaizu, T. Iwasaki, Y. Tsukahara, E. Tsuchida, Macromolecules 2004, 37, 1257.

[14] T. Okamoto, K. Kudoh, A. Wakamiya, S. Yamaguchi, Chem. Eur. J. 2007, 13, 548 .

[15] D. Wasserberg, S. C. J. Meskers, R. A. J. Janssen, E. Mena-Osteritz, P. Bäuerle, J. Am. Chem. Soc. 2006, 128, 17007.

[16] M. Rubio, M. Merchán, R. Pou-Amerigo, E. Ortí, ChemPhysChem 2003, 4, 1308.

[17] M. Rubio, M. Merchán, E. Ortí, ChemPhysChem 2005, 6, 1357.

[18] B. Milián Medina, D. Wasserberg, S. C. J. Meskers, E. Mena-Osteritz, P. Bäuerle, J. Gierschner, J. Phys. Chem. A 2008, 112, 13282.

[19] M. G. Hill, K. R. Mann, L. L. Miller, J. Penneau, J. Am. Chem. Soc. 1992, 114, 2728.

[20] M. G. Hill, J. Penneau, K. R. Mann, L. L. Miller, Chem. Mater. 1992, 4,1106 . 
[21] B. Zinger, K. R. Mann, M. G. Hill, L. L. Miller, Chem. Mater. 1992, $4,1113$.

[22] L. L. Miller, K. R. Mann, Acc. Chem. Res. 1996, 29, 417.

[23] Y. Yu, E. Gunic, B. Zinger, L. L. Miller, J. Am. Chem. Soc. 1996, $118,1013$.

[24] D. D. Graft, R. G. Duan, J. P. Campbell, L. L. Miller, K. R. Mann, J. Am. Chem. Soc. 1997, 119, 5888.

[25] P. Baeuerle, U. Segelbacher, A. Maier, M. Mehring, J. Am. Chem. Soc. 1993, 115, 10217.

[26] P. Bäuerle, U. Segelbacher, K. Gaudl, D. Huttenlocher, M. Mehring, Angew. Chem. 1993, 105, 125; Angew. Chem. Int. Ed. Engl. 1993, 32, 76.

[27] D. Fichou, B. Xu, G. Horowitz, F. Garnier, Synth. Met. 1991, 41, 463.

[28] J. V. Caspar, V. Ramamurthy, D. R. Corbin, J. Am. Chem. Soc. 1991, $113,600$.

[29] J. A. E. H. van Haare, E. E. Havinga, J. L. J. van Dongen, R. A. J. Janssen, J. Cornil, J. L. Brédas, Chem. Eur. J. 1998, 4, 1509.

[30] T. Satou, T. Sakai, T. Kaikawa, K. Takimiya, T. Otsubo, Y. Aso, Org. Lett. 2004, 6, 997.

[31] D. Yamazaki, T. Nishinaga, N. Tanino, K. Komatsu, J. Am. Chem. Soc. 2006, 128, 14470.

[32] J. Cornil, D. Beljonne, J. L. Brédas, J. Chem. Phys. 1995, 103, 842.

[33] D. Beljonne, J. Cornil, H. Sirringhaus, P. J. Brown, M. Shkunov, R. H. Friend, J. L. Brédas, Adv. Funct. Mater. 2001, 11, 229.

[34] M. Rubio, E. Ortí, R. Pou-Amerigo, M. Merchán, J. Chem. Phys. 2001, 105, 9788.

[35] T. Keszthelyi, M. M. L. Grage, J. F. Offersgaard, R. Wilbrandt, J. Phys. Chem. A 2000, 104, 2808.

[36] Y. Gao, C. G. Liu, Y. S. Jiang, J. Phys. Chem. A 2002, 106, 5380.

[37] S. S. Zade, M. Bendikov, J. Phys. Chem. B 2006, 110, 15839.

[38] U. Salzner, J. Chem. Theory Comput. 2007, 3, 1143.

[39] Z. Xu, D. Fichou, G. Horowitz, F. Garnier, J. Electroanal. Chem. 1989, 267, 339

[40] J. L. Brédas, J. Chem. Phys. 1985, 82, 3808.

[41] G. Macchi, B. Milián Medina, M. Zambianchi, R. Tubino, J. Cornil, G. Barbarella, J. Gierschner, F. Meinardi, Phys. Chem. Chem. Phys. 2009, 11, 984.

[42] R. Malavé Osuna, V. Hernández, J. T. López Navarrete, J. Aragó, P. M. Viruela, E. Ortí, T. Suzuki, S. Yamaguchi, J. T. Henssler, A. J. Matzger, ChemPhysChem 2009, 10, 3069.

[43] A. P. Scott, L. Radom, J. Phys. Chem. 1996, 100, 16502.

[44] D. Birnbaum, B. E. Kohler, J. Chem. Phys. 1991, 95, 4783.

[45] D. Birnbaum, B. E. Kohler, J. Chem. Phys. 1989, 90, 3506.
[46] D. Birnbaum, D. Fichou, B. E. Kohler, J. Chem. Phys. 1992, 96, 165 [47] J. Gierschner, H. G. Mack, H. J. Egelhaaf, S. Schweizer, B. Doser, D. Oelkrug, Synth. Met. 2003, 138, 311.

[48] Gaussian 03, Revision D.02, M. J. Frisch, G. W. Trucks, H. B. Schlegel, G. E. Scuseria, M. A. Robb, J. R. Cheeseman, J. A. Montgomery, Jr., J. Vreven, T.; , K. N. Kudin, J. C. Burant, J. M. Millam, S. S. Iyengar, J. Tomasi, V. Barone, B. Mennucci, M. Cossi, G. Scalmani, N. Rega, G. A. Petersson, H. Nakatsuji, M. Hada, M. Ehara, K Toyota, R. Fukuda, J. Hasegawa, M. Ishida, T. Nakajima, Y. Honda O. Kitao, H. Nakai, M. Klene, X. Li, J. E. Knox, H. P. Hratchian, J. B. Cross, C. Adamo, J. Jaramillo, R. Gomperts, R. E. Stratmann, O. Yazyev, A. J. Austin, R. Cammi, C. Pomelli, J. W. Ochterski, P. Y. Ayala, K. Morokuma, G. A. Voth, P. Salvador, J. J. Dannenberg, V. G. Zakrzewski, S. Dapprich, A. D. Daniels, M. C. Strain, O. Farkas, D. K. Malick, A. D. Rabuck, K. Raghavachari, J. B. Foresman, J. V. Ortiz, Q. Cui, A. G. Baboul, S. Clifford, J. Cioslowski, B. B. Stefanov, G. Liu, A. Liashenko, P. Piskorz, I. Komaromi, R. L. Martin, D. J. Fox, T. Keith, M. A. Al-Laham, C. Y. Peng, A. Nanayakkara, M. Challacombe, W. P. M. Gill, B. Johnson, W. Chen, M. W. Wong, C. Gonzalez, J. A. Pople, Gaussian Inc.: Pittsburgh PA, 2003.

[49] A. D. Becke, J. Chem. Phys. 1993, 98, 5648.

[50] C. Lee, W. Yang, R. G. Parr, Phys. Rev. B 1988, 37, 785.

[51] M. M. Francl, W. J. Pietro, W. J. Hehre, J. S. Binkley, M. S. Gordon, D. J. Defrees, J. A. Pople, J. Chem. Phys. 1985, 77, 3654.

[52] M. E. Casida, C. Jamorski, K. C. Casida, D. R. Salahub, J. Chem. Phys. 1998, 108, 4439.

[53] C. Jamorski, M. E. Casida, D. R. Salahub, J. Chem. Phys. 1996, 104, 5134.

[54] M. Petersilka, U. J. Grossmann, E. K. U. Gross, Phys. Rev. Lett. 1996, 76, 1212

[55] J. Tomasi, M. Persico, Chem. Rev. 1994, 94, 2027.

[56] C. S. Cramer, D. G. Truhlar, Solvent Effects and Chemical Reactivity, Kluwer, Dordrecht, 1996.

[57] A. E. Reed, F. Weinhold, J. Chem. Phys. 1983, 78, 4066.

[58] A. E. Reed, R. B. Weinstock, F. Weinhold, J. Chem. Phys. 1985, 83, 735.

[59] A. E. Reed, L. A. Curtiss, F. Weinhold, Chem. Rev. 1988, 88, 899.

[60] S. Portmann, H. P. Lüthi, Chimia 2000, 54, 766.

Received: December 5, 2009 Published online: March 31, 2010 